\title{
Characterisation of microRNAs regulated by vitamin D and lipid loading in immortalised hepatic stellate cells
}

\author{
Z. Zhang ${ }^{1}$, R. Moon ${ }^{1}$, H. Fuller ${ }^{1}$, X. Tan ${ }^{1}$, M.J. Holmes ${ }^{1}$, J.L. Thorne ${ }^{1}$ and J.B. Moore ${ }^{1}$ \\ ${ }^{1}$ School of Food Science and Nutrition, University of Leeds, Leeds, UK
}

Evidence for a role for vitamin D in metabolic-associated fatty liver disease (MAFLD) pathogenesis is conflicting ${ }^{(1)}$. The molecular pathogenesis of MAFLD is complex, involving numerous signalling molecules including microRNAs (miRNAs) ${ }^{(2)}$. Moreover, miRNAs play essential roles in mediating the cellular response to vitamin D, including the post-transcriptional regulation of the vitamin D receptor $(\mathrm{VDR})^{(3)}$. However, the potential roles for vitamin D regulated miRNAs in MAFLD pathogenesis has been relatively unexplored. With an overall objective of investigating the role of vitamin D regulated miRNAs in MAFLD, the aims of these experiments were to first measure miRNA expression in immortalised hepatic stellate cells (LX-2 cells) in response to vitamin D or lipid loading alone, or in combination; and then to examine potential functional and mechanistic effects using bioinformatics.

Cells were cultured for $16 \mathrm{hrs}$ in media with charcoal-stripped serum prior to $24 \mathrm{hrs}$ treatment with either $100 \mathrm{nM}$ 1 $\alpha, 25$-dihydroxyvitamin $\mathrm{D} 3$, or fatty acid $(500 \mu \mathrm{M}, 1: 1$ oleic acid: palmitic acid), or both in combination. miRNA samples from four independent experiments were collected using mirVANATM miRNA isolation reagents and cDNA synthesised. TaqMan ${ }^{\circledR}$ array human miRNA A+B cards were used to measure miRNA expression in equally pooled miRNA samples. Data were examined both by relative fold change and significance analysis of microarrays (SAM) approaches (in R version v4.0.3). Bioinformatic analyses included: the identification of miRNA gene targets, gene set enrichment analysis (GSEA), and functional annotation and pathway analyses; and were performed utilising the miRWALK (v3.0), DAVID (v6.8) knowledgebases and their associated tools.

768 miRNAs were measured across all four treatment groups. Of these, approximately $17 \%$ of detected miRNAs were found increased (fold change $>2.6$ ) and decreased (fold change $<0.67$ ) relative to vehicle-treated cells $(17.8 \%, 17.9 \%$ and $16.6 \%$ in vitamin $\mathrm{D}$, fatty acid, and co-treated cells respectively). However, SAM analysis across groups suggested 82 miRNAs as significantly modulated at delta $=1.211$ and a median false discovery rate of 0.2 . Utilising miRWALK and GSEA, 33 pathways were found significantly altered under all three different treatment conditions. These included biologically relevant pathways such as FoxO, mTOR, MAPK, p53, Ras, and TGF- $\beta$ (all $\mathrm{P}<0.001$ ).

In summary, these experiments identify miRNAs regulated by vitamin D and lipid loading in immortalised hepatic stellate cells. Bioinformatic results highlight signalling pathways with critical roles in MAFLD, diabetes, cardiovascular disease and cancer progression. Ongoing work is integrating these data with previous results of immortalised hepatocytes (HepG2) and literature evidence. From this, a subset of candidate miRNAs will be followed up for independent verification of functional and mechanistic effects in both vitamin D metabolism and MALFD progression.

\section{References}

1. Zhang Z, Thorne JL \& Moore JB (2019) Curr Opin Clin Nutr Metab Care 22, 449-458.

2. Wang X, He Y, Mackowiak B et al. (2020) Gut.

3. Zenata O \& Vrzal R (2017) Oncotarget 8, 35390-35402. 